\title{
Nanotechnology provides new methods for oral delivery of peptides
}

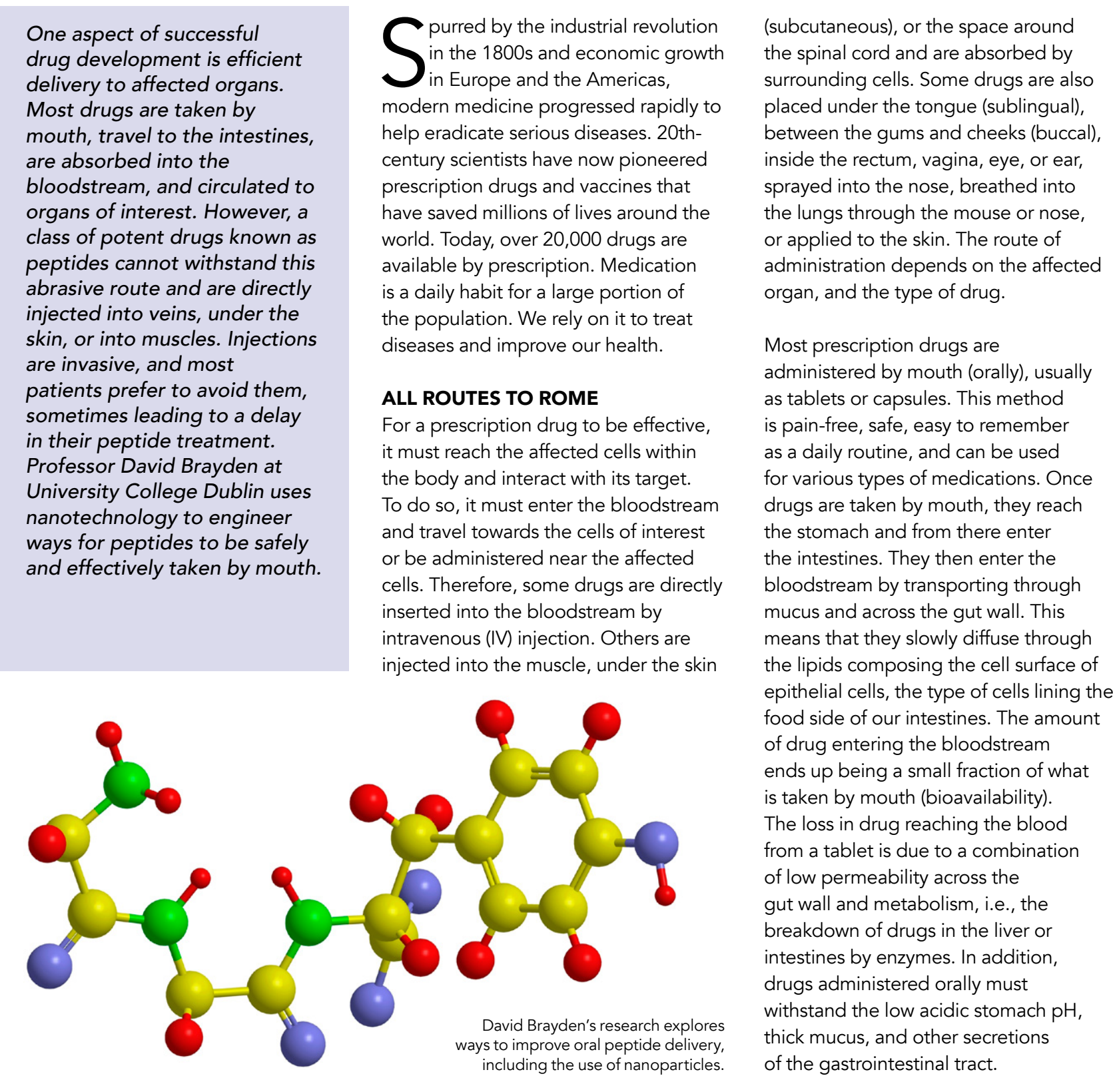

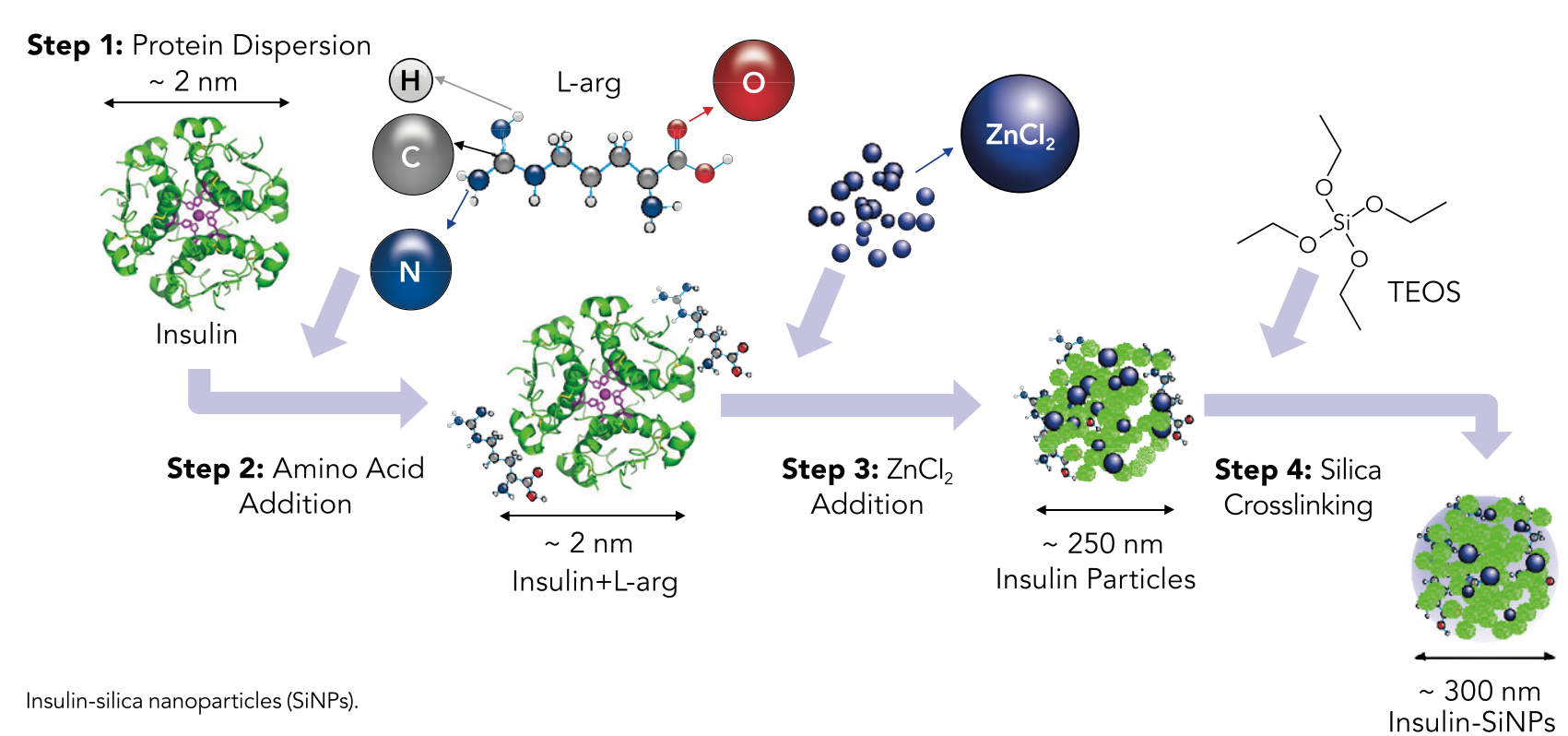

SPECIFICITY IS KEY

Small molecule drugs, i.e., drugs of approximately $1 \mathrm{~nm}$ in size that are
manufactured by chemical synthesis,

are the most prescribed medications

and they are typically delivered orally.

They can inhibit a protein that functions abnormally and is causing a disease. Small molecules are easy to manufacture reproducibly. However, they are often

for prostate cancer, and Bortezomib and Cartllzomib for multiple myeloma. is anticipated that approved peptide medications will steadily increase in coming years.

ROADBLOCK FOR PEPTIDES Peptides are particularly susceptible to breakdown by first-pass metabolism.
They also tend to be too large to be additional proteins

are designed to target. This can lead to unwanted side-effects.

Oral delivery of peptides will help by enzymes, such as peptidases or $\mathrm{pH}$ adfiers. Peptidase enzyme inhibitors with agents that facilitate permeability across the gut, known as chemical permeation enhancers (PEs). This combination of agents protects against enzymes, overcomes mucus and low $\mathrm{pH}$, while PEs can be released at the same time as peptides in the intestine when they are present in enteric-coated tablets or capsules. The most commonly patients avoid inconvenient injections, salcaprozate sodium S, (SNAC) and sodium encourage better adherence to therapy, caprate $\left(\mathrm{C}_{10}\right)$. They $\begin{aligned} & \text { Another group of } \\ & \text { drugs, known as }\end{aligned} \quad$ and will help them to opt for these tested in clinical trials
and SNAC is present drugs, known as molecules earlier in their disease. a in recently marketed
peptides, have excellent specificity
to their target protir tablet of the peptide, to their target proteins. Peptides are absorbed through the intestine wall therapeutic intervention by peptides, If administered orally, the amount of mimics a natural process in the cells by woptide passing through to the blood replacing a hormone or enzyme whose $\quad 95 \%$ of peptides are delivered by IV or levels are abnormally diminished inside subcutaneous injection. Unfortunately, cells. Thanks to this property, peptide most patients find injections painful and drugs are also safe and effective. time-consuming and would avoid them if they could.

The use of peptides as therapeutics was introduced in the 1920s, when insulin was manufactured as a drug for type I diabetes. Since then, they have constituted life-saving 20th century medicines. Over 60 peptide drugs are approved for safe use in humans. Examples include insulin and exenatide

\section{ORAL TABLET}

VERSIONS OF PEPTIDES Scientists have attempted to solve this problem by enclosing peptides within capsules to protect hem from low pH and to deliver them to the right part specific inhibe. They can also add of $<1 \%$. The effects of these PEs on epithelial cells lining the intestine walls have been investigated for over three decades. What is still lacking is our knowledge of exactly how these PE agents work.

As Professor of Advanced Drug Delivery Dr David Brayden at University College into identifying the methods of action the two agents over 20 years ago. $C_{10}$ is of epithelial cells and opening surface that connace diand opents that connect adjacent cells (known as
tight junctions) to facilitate peptide Dublin has devoted significant resources of PEs. He and his team first studied 


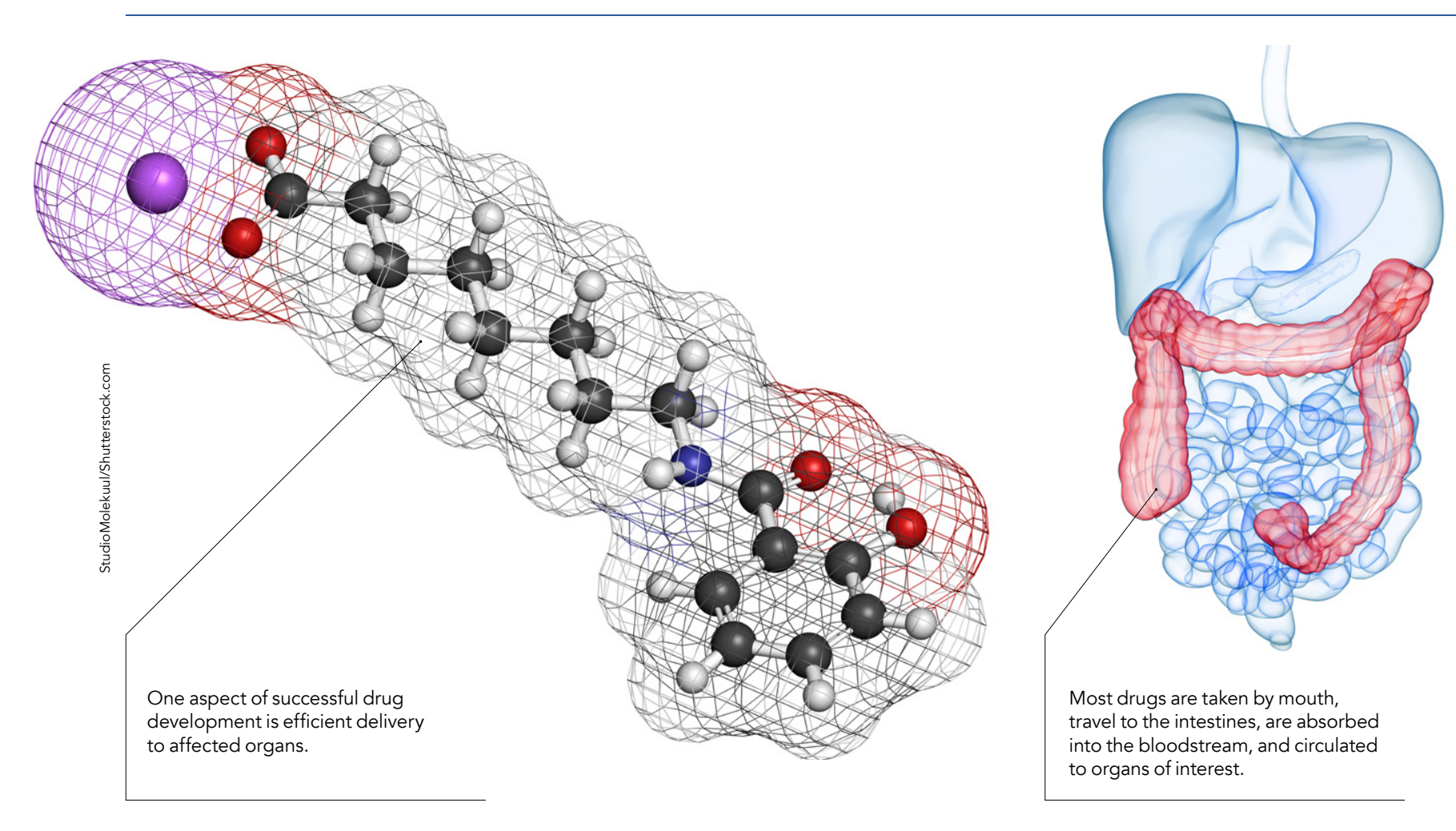

crossing. On the other hand, SNAC is thought to increase passive transport of peptides across epithelial cells by changing peptide solubility. SNAC is also thought to reduce levels of acid in the stomach in the immediate region around the semaglutide tablet, and to inhibit stomach enzymes (e.g., pepsin) that would break it down.

Both PE agents are considered safe, but they are yet to be studied for periods longer than six months. Prof Brayden's team performed their own SNAC experiments to confim that $\mathrm{C}_{10}$ and SNAC are not toxic to cultured gut cells, never be used in the clinic At close examination, both agents made the cell membrane more permeable, but also altered the flow of calcium ions in and out of cells and disturbed metabolism. According to Prof Brayden, $\mathrm{C}_{10}$ is more potent than SNAC but their efficacy is similar. Nevertheless, in his hands, both agents acted with several features suggesting some common mechanisms relating to detergent action. Both PEs only marginally increased absorption of peptides from the gut into the bloodstream, however hat may be enough for some candidates since peptides can be very potent drugs. The lack of biological reasons to pref one PE agent over another, points
Overall, nanotechnology can enable peptides to gain at least some access to the bloodstream by the oral route.

synthesised and manufactured with peptides in tablets, as well as takin into account commercial aspects before being used in the clinic.

BIG SOLUTIONS FROM TINY PARTICLES

Most current solutions for creating that tablet versions of peptides are effective option might be the A mo nanoparticles. These are materials whose size ranges from 1 to 1,000 nanometres. That is a billionth of a meter, or 10,000-times smaller than the thickness of a single hair. Nanoparticles have a wide range of uses, from transparent sunscreens food packaging. Their small size also makes them ideal for penetrating mucus and accessing the gut wall to deliver peptides to the bloodstream But they are still under development for this function, and not widely tested in clinical trials.

Prof Brayden's team are dedicated to developing a new type of nanoparticle for oral peptide delivery to help patent

Overall, nanotechnology can enable earlier in their disease, and better adhere to their dosing instructions. The researcher's nanoparticles are coated with common silica which successfully encapsulates insulin in the core, delivering higher amounts from the gut to the bloodstream. The nanoparticles did not cross the gut wall de delivered il tse enough to wall, the help of other components in the particle. The manufacturing process for scalable to quantities needed for the clinic. This work represents a new type of nanoparticle, with many advantages over its predecessors. Notably, the Irish team expects that their research will have additional practical applications, such as local delivery of medication to arthritic joints. peptides to gain at least some access to the bloodstream by the oral route, pate of progress towards treatment of having a major impact on hum drugs, these nanoparticles is reproducible and tholds the potential to increase the

\section{g Behind the Research Prof David Brayden}

\section{Research Objectives}

David Brayden's research explores ways to improve ord peptide delivery, including the use of nanoparticles.

\section{Detail}

Professor David Brayden, Room 231, Veterinary Sciences Centre, University College Dublin, Belfield,

Bio

David Brayden is Full Professor of Advanced Drug Delivery at the Schoo Dublin (UCD), and a Senior Fellow of the UCD Conway Institute. He is a pharmacologist and spent 10 years as a senior scientist at Elan Pharmaceuticals. He is the author/ co-author of more than 200 research publications in drug delivery, and is a co-lead Principal Investigator in the SFI CURAM Centre for Medical Devices. He is a Fellow of the Controlled Release Society and the American Association of Pharmaceutical Scientists.

\section{Funding}

Science Foundation Ireland CURAM Center for Medica Devices (www.curamdevices.ie ); EU FP7 TRANS-INT;

\section{Collaborators}

- Prof Elias Fattal (Galien Institute, Paris) (Sanofi Recherche, Paris)

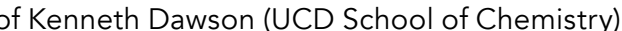

- PhD student Caroline Twarog (UCD)

- Postdocs: Dr Fiona McCartney (UCD). Dr Delyan

Hristov (University of Maryland, USA)

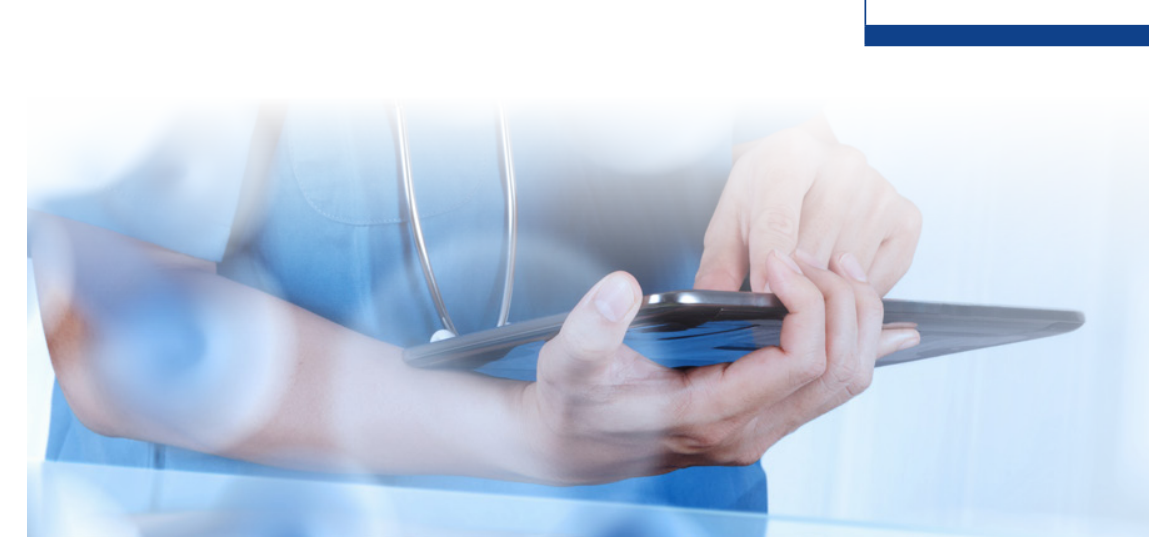

\section{References}

Twarog, C., Fattah, S., Heade, J., Maher, S., Fattal, E., Brayden, DJ. (2019) Intestinal Permeation Enhancers for Oral Delivery of Macromolecules. A Comparison between Salcaprozate Sodium (SNAC) and Sodium Caprate (C10). Pharmaceutics, 11(2). doi:10.3390/pharmaceutics11020078

Hristov, D., McCartney, F., Beirne, J., Mahon, E., Reid, S., Bhattacharjee, S., Penarier, G., Werner, U., Bazile, D., Brayden, DJ. (2020) Silica-Coated Nanoparticles with a Delivery. ACS Appl. Mat. 12(1):1257-1269. do:101021/ acsami.9b16104

Twarog, C., Fattal, E., Liu, K., O'Brien, P.J., Dawson, KA., Illel, B., Brayden, DJ. (2020) Comparison of the mechanisms of action of the intestinal permeation enhancers SNAC and sodium caprate in Caco-2 assays. Eur. J. Pharm. Biopharm (Under review)

\section{Personal Response}

How soon do you anticipate silica-coated ic to deliver

II A programme to test the nanoparticles entrapping dosed to large animals, including pigs, would be the next milestone. Since all the materials in the particle have a history of use in humans, the toxicology testing should be relatively straightforward. A target of reaching a first-in-human Phase I trial in 3-4 years with Parting an be achieved. 